\title{
Early Repair of Sternal Instability Prevents Mediastinitis
}

\author{
Koray Aykut $^{1 *}$, Gokhan Albayrak ${ }^{1}$, Aycan Kavala ${ }^{2}$, Mehmet Guzeloglu', \\ Kemal Karaarslan ${ }^{3}$, Eyup Hazan ${ }^{1}$ \\ ${ }^{1}$ Department of Cardiovascular Surgery, Izmir University, Izmir, Turkey \\ ${ }^{2}$ Department of Cardiovascular Surgery, Bakirkoy Dr. Sadi Konuk Research Hospital, Istanbul, Turkey \\ ${ }^{3}$ Department of Cardiovascular Surgery, Tepecik Research Hospital, Izmir, Turkey \\ Email: ${ }^{\text {drkaykut@hotmail.com }}$
}

Received December 1, 2013; revised December 31, 2013; accepted January 8, 2014

Copyright (C) 2014 Koray Aykut et al. This is an open access article distributed under the Creative Commons Attribution License, which permits unrestricted use, distribution, and reproduction in any medium, provided the original work is properly cited. In accordance of the Creative Commons Attribution License all Copyrights (C) 2014 are reserved for SCIRP and the owner of the intellectual property Koray Aykut et al. All Copyright (C) 2014 are guarded by law and by SCIRP as a guardian.

\section{ABSTRACT}

Background: Discharge due to superficial wound infection and sternal dehiscence are the common complications of median sternotomy. This condition leads to mediastinitis. Mediastinitis is a serious condition which increases morbidity, mortality and hospital costs. The correct time for surgical treatment is contraversial in cases who have sternal dehiscence and discharge due to superficial infection. In this study, patients received sternal stabilization as soon as a diagnosis was made and monitored for the development of mediastinitis. Methods: Among the 1440 patients who had undergone elective CABG, a total of 85 patients who developed sternal dehiscence and superficial wound discharge were included in this study. These patients were re-operated on as soon as diagnosed and early sternal stabilization was provided. Results: The ratio of overall dehiscence was $5.9 \%$. Mediastinitis developed in only 2 of the 85 patients. These patients were treated with VAC (Vacuum Assisted Closure) therapy. The ratio of overall mediastinitis was 0.13. Mediastinitis-related mortality was not detected. Conclusion: The ratio of mediastinitis decreases when patients who have discharge due to sternal dehiscence and superficial wound infection are immediately reviewed without waiting for the results of conventional methods such as antibiotic treatment and chest binder support.

\section{KEYWORDS}

Coronary Artery Bypass Grafting; Sternal Dehiscence; Mediastinitis

\section{Introduction}

Approximately 700,000 open heart procedures are performed every year in the United States and of these, more than $67 \%$ are coronary artery bypass grafting procedures. Median sternotomy represents the main approach in cardiac surgery as it allows access to all important structures of the heart. Sternal dehiscence which is defined as the separation of the bony sternum, is an infrequent but serious complication that is often a precursor to mediastinitis. Mediastinitis is a severe complication of cardiac surgery and is also associated with serious mortality and cost $[1,2]$. It is a devastating infectious complication, involving the mediastinal space of the chest. The literature reports mediastinitis rates following cardiac surgery

"Corresponding author. of between $0.5 \%$ and $5 \%$, with a mortality rate as high as $40 \%[3,4]$.

The correct time for surgical intervention is contraversial in patients who have discharge from the wound due to sternal instability and superficial wound infection in the early period following open heart surgery. Usually, the initial treatment of discharge is by the administration of broad spectrum antibiotics and the recovery of the sternum is provided with chest binder support. However, some of these patients develop mediastinitis. In this study, patients who developed (serosanguinous or purulent) discharge at the wound site, in addition to sternal dehiscence in the early period following coronary artery bypass, were immediately evaluated and they underwent reoperation for sternal stabilization. Patients were monitored for mediastinitis development and the results were reported. 


\section{Patients and Methods}

We reviewed data on 1440 consecutive patients who underwent elective coronary artery bypass surgery between 2010 and 2013. Among these patients, 85 (5.90\%) had experienced superficial wound infection (serosangineous or purulent discharge from the skin without fever) and sternal dehiscence within 2 weeks after the operation. It's the period including in-hospital stay and the first week check-up following cardiac surgery. Although we did not have any, patients who were considered to have mediastinitis when sternal dehiscence was detected, were excluded. Also, redo operations, sternal fractures and reexploration for bleeding were the other exclusion criteria.

Post-sternotomy mediastinits was defined according to the guidelines of the Centers for Disease Control and Prevention (CDC). Diagnosis required at least one of the following criteria: 1) an organism was isolated from cultures of mediastinal tissue or fluid obtained during surgery or needle aspiration; 2) evidence of mediastinitis was seen during operation; or 3) chest pain, sternal instability, or fever $\left(>38^{\circ} \mathrm{C}\right)$ was present in addition to purulent discharge from the mediastinum or an organism that was isolated from blood cultures or cultures of drainage from the mediastinal area.

During bypass surgery, the same sternal closure technique was applied in all patients. Before surgery and after cleaning with $10 \%$ povidone iodine solution, the skin was covered with polyurethane drapes. Bone wax was never used for hemostasis because of its negative effect on wound healing. The sternum was closed using No.6 steel wires. The wires were passed through the sternum approximately 1 to $1.5 \mathrm{~cm}$ lateral to the midline. The 2 free ends of the wire were pulled and then crossed in a figure-of-8 configuration. Using a rotary movement of the wrist along with a vertical pull on the wires, the wires were twisted tightly until the 2 bone edges approximated. Four figure-of-eight sutures were used in all patients (Figure 1). Cefazolin 1 gr. was administered within 30 min before the initial incision and was continued for $72 \mathrm{~h}$ postoperatively as antibiotic prophylaxis.

Sternal weave closure technique (Robicsek) was performed for 85 patients who had sternal instability in addition to superficial wound infection with serosangineous or purulent discharge from the skin without fever. A pericostal weave was achieved by passing the wire through the intercostal spaces anteriorly and posteriorly in an alternating sequence up to the xiphoid process. The same wire was reversed to pass through the intercostal spaces in the reverse direction from the xiphoid process up to the manubrium. The free ends of the wire on the anterior plane of the manubrium were twisted. Two transmanubrial and 4 - 6 parasternal single wire loops were placed around the bilateral pericostal weave (Figure 2). Antibiotic treatmet was given according to antibiogram results.

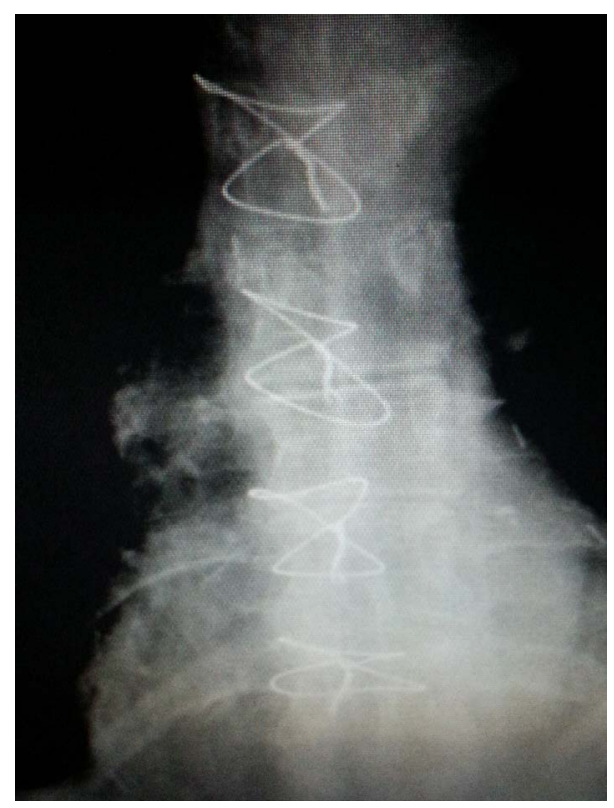

Figure 1. Figure-of-eight closure of median sternotomy.

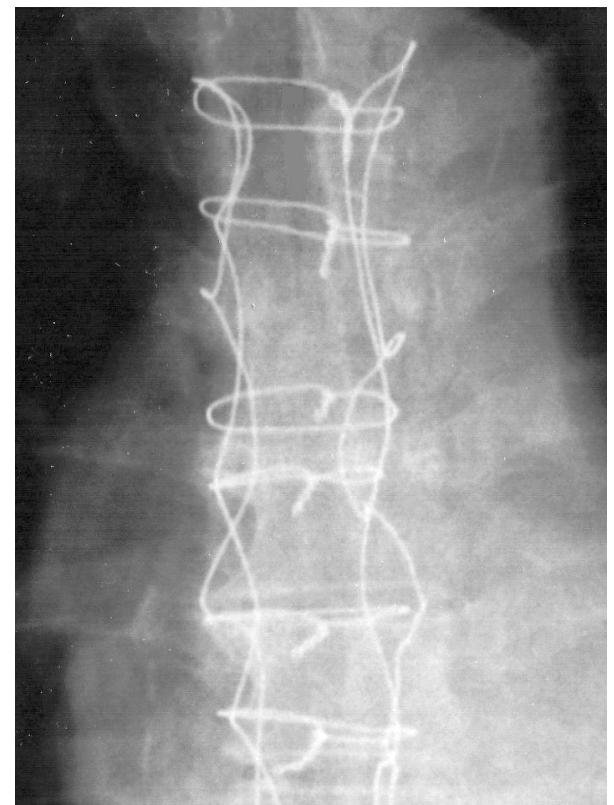

Figure 2. Pericostal weave closure of median sternotomy.

All patients were given chest binder support and advised to move carefully so that strain was not put on the sternum.

\section{Results}

The demografic profile and risk factors of the study group (n: 85) were shown in Table 1 . Diabetes mellitus, obesity and smoking were the most common comorbidities among the patients with sternal instability.

Obesity was defined as body mass index $>30 \mathrm{~kg} / \mathrm{m}^{2}$. Renal failure was defined as a postoperative serum crea- 
Table 1. Demographic profile and risk factors of the patients.

\begin{tabular}{cc}
\hline Variable & Study group \\
\hline No. of patients & 85 \\
Mean age (years) & $57.4 \pm 5.3$ \\
Female & $39(45.8 \%)$ \\
Smoking & $51(60.0 \%)$ \\
COPD & $15(17.6 \%)$ \\
Obesity & $47(55.2 \%)$ \\
Diabetes mellitus & $62(72.9 \%)$ \\
Hypertension & $45(52.9 \%)$ \\
Fragile sternum & $35(41.1 \%)$ \\
Renal failure & $2(2.3 \%)$ \\
Prolonged respiratory support & $3(3.5 \%)$ \\
LIMA harvest & $70(96.4 \%)$ \\
Mean no. of bypass grafts & $3.48 \pm 0.4$ \\
Mean CPB time & $61.4 \pm 8.6$ \\
\hline
\end{tabular}

tinine level greater than $1.5 \mathrm{mg}$ in a patient with previously normal kidney function. Diagnosis of COPD was considered in any patient who had symptoms of a chronic cough, sputum production, and dyspnea. Diagnosis was confirmed by spirometry (forced expiratory volume in the first second of expiration/forced vitalcapacity $<70 \%$ ).

Of the 85 swabs taken, 82 were culture positive. Staphylococcus epidermidis was the causative organism in 55.2 of cases. The other isolates were Staphylococcus aureus, Escherichia coli, Streptococcus pneumoniae and Pseudomonas aeruginosa. The distribution is shown in Table 2. Antimicrobial therapy was planned due to antibiograms.

Two patients experienced mediastinitis caused by Staphylococcus aureus isolates which were sensitive to teicoplanin. Both of them were diabetic obese patients. They were treated with vacuum-assisted closure (VAC). The therapy unit delivered a continuous negative pressure of 100 - $125 \mathrm{mmHg}$. The VAC dressings were changed twice a week. The criteria for VAC removal were, negative bacterial culture, absence of fever, falling C-reactive protein levels and clinically healthy granulating wound. Both patients recovered in a few weeks with long-term antibiotics therapy.

Overall sternal dehiscence rate was 5.90\%. Mediastinits rate was found $0.13 \%$ and mortality due to mediastinits was not detected (Table 3).

\section{Discussion}

Sternotomy provides excellent access to the heart and great vessels since it was first introduced by Julian et al. in 1957 [5]. However it is associated with many complications such as sternal dehiscence and mediastinitis that cause tremendous morbidity, mortality and cost.
Table 2. Causative organisms.

\begin{tabular}{ccc}
\hline Organism & No. & \% \\
\hline Staphylococcus epidermidis & 47 & 55.2 \\
Staphylococcus aureus & 23 & 27.0 \\
Escherichia coli & 9 & 10.5 \\
Streptococcus pneumoniae & 2 & 2.3 \\
Pseudomonas aeruginosa & 1 & 1.2 \\
Unknown & 3 & 3.5 \\
\hline
\end{tabular}

Table 3. Post-operative sternotomy complications.

\begin{tabular}{ccc}
\hline & No. & \% \\
\hline Total no. of patients & 1440 & - \\
Sternal instability & 85 & 5.90 \\
Mediastinitis & 2 & 0.13 \\
Mortality due to mediastinitis & 0 & - \\
\hline
\end{tabular}

The optimal technique for sternal closure is unclear. Simple interrupted sutures, figure-of-eight closure, ory any other technique can be used in routine cases. It depends on the surgeon's preference and experience. Although reinforced closure techniques are less attractive options for routine closure because they are considered to be time-consuming and to have a high risk for bleeding, some authors suggest that prophylactic sternal weave closure is more beneficial for diabetic obese patients undergoing CABG [3].

Casha and colleagues calculated that the force generated during coughing is approximately $150 \mathrm{~kg}$, and they recommended that at least 6 simple interrupted wires should be used for sternal closure [6]. In our study, we used 4 figure-of-eight sutures in all cases. In patients who underwent reoperation for revision of sternal instability, we used 8 interrupted wires (2 transmanubrial and 6 parasternal) around a continuous vertical pericostal weave.

Risk factors for mediastinitis are off-midline sternotomy, osteoporosis, prolonged cardiopulmonary bypass, transverse fractures of the sternum, bilateral internal mammary artery harvesting, chronic obstructive pulmonary disease, redo operations, reanl failure, diabetes mellitus, chronic steroid use, obesity, concurrent infection and prolonged respiratory [7]. Diabetes mellitus, obesity and smoking were the most common comorbidities among the patients with sternal instability in our study. Also, the patients who experienced mediastinitis had diabetes mellitus and obesity.

In 1963 Shumacker and Mandelbaum described a method for the treatment of postoperative mediastinitis [8]. Their approach included surgical debridement, insertion of a drainage system with continuous irrigation and primary wound closure. Some centers still use this conventional therapy.

The vacuum-assisted closure (VAC) was introduced in 
1997 and combines the advantages of both open and closed treatment. Besides an improved local perfusionand oxygenation, the quantitative bacterial florais rapidly reduced and the formation of scar tissue is stimulated. Several studies showed the benefits of a VAC therapy in terms of shorter hospitalizations, earlier secondary wound closure and a lower mortality compared to conventional therapy $[9,10]$. We used VAC therapy to treat our patients with mediasitinitis. They recovered well in a few weeks.

In our study, the ratio of overall sternal dehiscence was found as $5.9 \%$, which is consistent with literature. However, early sternal stabilization was performed when discharge was seen, so the ratio of mediastinitis was lower when compared to that found in literature.

\section{Conclusion}

Sternal instability leads to mediastinitis when it coexists with superficial wound infection. When such a condition is detected in the early post-operative period of patients who have had open heart surgery, the patient should be evaluated immediately without losing any time with chest binder support or antibiotic therapy. The ratio of mediastinitis significantly decreases in patients that have early sternal stabilization.

\section{REFERENCES}

[1] C. Diez, D. Koch, O. Kuss, R. E. Silber, I. Freidrich and J. Boergermann, "Risk Factors for Mediastinitis after Cardiac Surgery-A Retrospective Analysis of 1700 Patients," Journal of Cardiothoracic Surgery, Vol. 20, No. 2, 2007, p. 23. http://dx.doi.org/10.1186/1749-8090-2-23

[2] C. S. Hollenbeak, D. M. Murphy, S. Koenig, R. S. Woodward, W. C. Dunagan and V. J. Fraser, "The Clinical and Economic Impact of Deep Chest Surgical Site Infections Following Coronary Artery Bypass Graft Surgery,” Chest, Vol. 118, No. 2, 2000, pp. 397-402. http://dx.doi.org/10.1378/chest.118.2.397
[3] K. Aykut, B. Celik and U. Acıkel, "Figure-of-Eight versus Prophylactic Sternal Weave Closure of Median Sternotomy in Diabetic Obese Patients Undergoing Coronary Artery Bypass Grafting," Annals of Thoracic Surgery, Vol. 92, No. 2, 2011, pp. 638-641. http://dx.doi.org/10.1016/j.athoracsur.2011.04.034

[4] F. Ceresa, G. Casablanca and F. Patanè, "Complicated Sternal Dehiscence Treated with the Strasbourg Thoracic Osteosyntheses System (STRATOS) and the Transposition of Greater Omentum: A Case Report," Journal of Cardiothoracic Surgery, Vol. 5, 2010, p. 53. http://dx.doi.org/10.1186/1749-8090-5-53

[5] M. L. Dalton, S. R. Connally and W. C. Sealy, "Julian's Reintroduction of Milton's Operation,” Annals of Thoracic Surgery, Vol. 53, No. 3, 1992, pp. 532-533. http://dx.doi.org/10.1016/0003-4975(92)90293-D

[6] A. R. Casha, L. Yang, P. H. Kay, M. Saleh and G. J. Cooper, "A Biomechanical Study of Median Sternotomy Closure Techniques," European Journal Cardio-Thoracic Surgery, Vol. 15, No. 3, 1999, pp. 365-369. http://dx.doi.org/10.1016/S1010-7940(99)00014-7

[7] The Parisian Mediastinitis Study Group, "Risk Factors for Deep Sternal Wound Infection after Sternotomy: A Prospective Multicenter Study," Journal of Thoracic and Cardiovascular Surgery, Vol. 111, No. 6, 1996, pp. 12001207. http://dx.doi.org/10.1016/S0022-5223(96)70222-2

[8] H. B. Shumacker Jr. and I. Mandelbaum, "Continuous Antibiotic Irrigation in the Treatment of Infection," Archives of Surgery, Vol. 86, No. 3, 1963, pp. 384-387. http://dx.doi.org/10.1001/archsurg.1963.01310090034006

[9] R. J. Vos, A. Yilmaz, U. Sonker, J. C. Kelder and G. T. Kloppenburg, "Vacuum-Assisted Closure of Post-Sternotomy Mediastinitis as Compared to Open Packing," Interactive CardioVascular and Thoracic Surgery, Vol. 14, No. 1, 2012, pp. 17-21. http://dx.doi.org/10.1093/icvts/ivr049

[10] J. Sjögren, J. Nilsson, R. Gustafsson, M. Malmsjö and R. Ingemansson, "The Impact of Vacuum-Assisted Closure on Long-Term Survival after Post-Sternotomy Mediastinitis,” Annals of Thoracic Surgery, Vol. 80, No. 4, 2005, pp. 1270-1275.

http://dx.doi.org/10.1016/j.athoracsur.2005.04.010 